\title{
LA DIMENSIONE IMMAGINARIA DEL REALE SECONDO LA TEORIA DELLA COMPLESSITÀ DI PAVEL A. FLORENSKIJ $(1882-1937)^{1}$
}

\author{
The Imaginary Dimension of the Real According \\ to the Theory of Complexity by Pavel A. Florensky \\ $(1882-1937)$
}

Lubomír Žák

DOI: 10.17846/CL.2021.14.2.191-204

\begin{abstract}
K, Lubomír. The Imaginary Dimension of the Real According to the Theory of Complexity by Pavel A. Florensky (1882 - 1937). Having for many years the theme of "complex thought" as developed by Pavel Florensky at the centre of his philosophical and theological research interest, the author of the article highlights an important aspect of complexity, namely, the imaginary dimension as a structural part of reality. First of all, he shows how Florensky, famous Russian mathematician, physicist, electro-technical engineer, philosopher and theologian, comes to explain the existence of the imaginary dimension starting from his original interpretation of Albert Einstein's theory of relativity, made with reference to Dante Alighieri's Divine Comedy. Consequently, he explains that the existence of this dimension coincides with the existence of a general predisposition of every human being to the experience of the "imaginary" as a door towards the "mysterious depths" of reality, the depths that in many cases tend to manifest themselves. Christian theology and spirituality help to know and experience exactly this kind of complexity of reality, glimpsing its vital and dynamic connection with the Trinitarian being of God.
\end{abstract}

Keywords: Russian thought, theory of complexity, imaginary space, Dante Alighieri, Albert Einstein, general relativity, faith and raison

\section{Verso una visione globale del reale - in dialogo con A. Einstein}

Una grande competenza scientifica e una singolare ingegnosità di pensiero sono attributi ormai ben noti di Pavel Aleksandrovič Florenskij, versatile studioso delle discipline sia umanistiche che scientifiche, in particolare della filosofia, della teologia, della filologia, della teoria dell'arte, della matematica, della geometria, della fisica, della biologia, della mineralogia e della chimica. E va sempre ricordato che egli è riuscito a sviluppare i suoi straordinari talenti intellettuali e a farli fruttificare nonostante le difficili condizioni di vita e di lavoro in cui vennero a trovarsi in Russia, dopo la rivoluzione dottobre del 1917, tutti gli scienziati e i pensatori giudicati dal nuovo regime insidiosi e poco affidabili. In una lettera del 13 maggio 1937, scritta dal gulag staliniano a mezz'anno

1 Questo articolo è stato sostenuto dalla borsa di studio dell'Università Palacký di Olomouc: "Le ricerche neuroscientifiche come sfida per ripensare il contenuto e il linguaggio dell'antropologia e dell'etica cristiane" (IGA_CMTF_2020_003). 
dalla fucilazione, revisionando il suo operato, Florenskij stila un lungo elenco degli ambiti e delle questioni scientifiche più importanti di cui si è occupato con interesse e successo:

"In matematica: 1) I concetti matematici come elementi costitutivi della filosofia (discontinuità, funzioni ecc.). 2) La teoria degli insiemi e la teoria delle funzioni delle variabili reali. 3) Gli immaginari geometrici. 4) L'individualità dei numeri (numero-forma). 5) Lo studio delle curve in concreto. 6) I metodi di analisi della forma. In filosofia e storia della filosofia: 1) Le radici cultuali delle origini della filosofia. 2) La base cultuale e artistica delle categorie. 3) Le antinomie della ragione. 4) Lo studio storico-filologico-linguistico della terminologia. 5) Le basi materiali dell'antropodicea. 6) La realtà dello spazio e del tempo. In critica d'arte: 1) I metodi di descrizione e datazione degli oggetti dellarte antica russa (intaglio, articoli di gioielleria, pittura). 2) La spazialità nelle opere d’arte, in specie nelle arti figurative. In elettrotecnica: 1) Lo studio dei campi elettrici. 2) I metodi dell'analisi dei materiali elettrici: la base della scienza dei materiali elettrici. 3) Il significato delle strutture dei materiali elettrici. 4) La diffusione delle resine sintetiche. 5) La diffusione e l'elaborazione degli elementi della depolarizzazione aerea. 7) Le classificazioni e la standardizzazione di materiali, elementi ecc. 8) Lo studio dei minerali di carbonio come gruppo. 9) Lo studio di una serie di rocce. 10) Lo studio sistematico della mica e la scoperta della sua struttura. 11) Lo studio di suoli e terreni. E così via. Sono poi a parte: la fisica del gelo; l'uso delle alghe" (Florenskij 1998, 702; 2011, 399).

Sicuramente colpisce, quando si entra in contatto con il pensiero e le opere di Florenskij, non solo l'enciclopedismo delle conoscenze, ma soprattutto una forma mentis assolutamente polifonica, capace di orchestrare - all'interno di un unico quadro di pensiero - temi e prospettive notevolmente differenti, istaurando tra di essi "sia 'sequenze orizzontali', cioè sviluppi argomentativi, sia agganci verticali, cioè relazioni tra vari piani e livelli” (Tagliagambe 2006, 15). Il senso di tale orchestrazione è intimamente connesso con quello che egli considerò lo scopo principale della sua vita: "l'apertura di nuove vie per una futura e globale visione del mondo" (Florenskij 1994, 38; 2007a, 5), in grado di cogliere e descrivere la complessità del reale. ${ }^{2}$ Va sottolineato che Florenskij, riconoscendo nella matematica il primo e indispensabile presupposto di una futura visione del mondo, s'impegnò nell'ambiziosa impresa di prospettarla con la consapevolezza di dover rifiutare il dogmatismo di teorie scientifiche preconfezionate e di assumere, come punto di partenza e di costante verifica della sua ricerca, lo sperimento, ossia l'esperienza concreta di un vivo contatto con l'oggetto delle proprie indagini scientifiche. Erano, infatti, l'attenta osservazione dei fenomeni della natura e un certosino lavoro in laboratori forniti di indispensabili strumenti di analisi e di misurazione le sue attività congeniali e preferite, che gli furono possibili persino negli ultimi anni di vita trascorsi nel gulag.

Occorre tener presente che già da studente presso la Facoltà di Matematica e Fisica dell'Università di Mosca e fino all'arresto, nel 1933, Florenskij fu in stretto contatto con eminenti scienziati russi, unitamente ai quali seguì costantemente i più recenti sviluppi soprattutto della matematica e della fisica. Il che spiega come mai sia stato uno dei primi, in Russia, a leggere e a commentare le opere di Georg Cantor, e anche come mai non gli sia sfuggita la pubblicazione delle traduzioni russe degli studi dedicati al principio della relatività, inclusi quelli dello stesso Albert Einstein. Riguardo al fisico tedesco, insignito del premio Nobel per la fisica (1921), va rilevato che Florenskij aveva menzionato, seppur soltanto di sfuggita, il suo principio della relatività già nel 1915, nel

2 Tra gli studi dedicati in Italia alla "concezione globale del mondo" (al "pensiero complesso") di Florenskij si rimanda a Žák 2017; Guerrisi 2017; Tagliagambe - Spano - Oppo 2018; Burzo 2018. 
saggio Il significato dell'idealismo, dove aveva proposto una significativa riflessione sulla quarta coordinata del reale. E anche se, successivamente, non elaborò mai una monografia dedicata interamente a un approfondimento scientifico del principio della relatività, egli fece riferimento ad esso, direttamente o indirettamente, in diversi scritti. Lo citò ad esempio nel 1918 nel contesto delle lezioni sul culto, tenute per gli studenti dell'Accademia teologica di Mosca; e ne parlò successivamente nellopera filosofico-teologica Iconostasi (1921), ${ }^{3}$ nella parte dedicata al tema del tempo capovolto. Questo in qualche modo dimostra che tra gli impulsi che stimolarono Florenskij nel proseguire sulla via dell'elaborazione del "pensiero complesso" va annoverato anche quello offerto da Einstein, sebbene i due geni mai si siano incontrati di persona.

Esiste tuttavia uno scritto, del 1922, in cui Florenskij non solo cita, insieme al nome di Einstein, il principio speciale e quello generale della relatività, ma ne propone un approfondimento del tutto insolito e originale, che destò un vivo interesse tra molti intellettuali russi dell'epoca, sia matematici e fisici che filosofi e letterati, provocando, al contempo, una feroce critica da parte degli scienziati collocati nell'orbita dell'ideologia del nuovo regime. Mi riferisco al breve ma speculativamente intenso opuscolo di geometria analitica Gli immaginari in geometria. L'ampliamento dell'ambito delle immagini geometriche bidimensionali (Tentativo di una nuova interpretazione degli immaginari) (Florenskij 1991; 2007b). ${ }^{4}$ Se Florenskij ha la fama di essere interprete della teoria della relatività generale di Einstein, l'ha guadagnata soprattutto grazie a questo testo. Eppure, con esso, non si spense il suo interesse per la teoria della relatività generale, che ad esempio adoperò, come una delle prospettive teoretiche di riferimento, nel contesto delle lezioni tenute negli anni 1923 1925 agli Atelier superiori tecnico-artistici di Stato e pubblicate, dopo la sua morte, con il titolo L'analisi della spazialità (e del tempo) nelle opere d'arte figurativa. I richiami espliciti e impliciti al principio della relatività generale sono, peraltro, presenti anche nel saggio Prospettiva rovesciata e soprattutto nelle riflessioni sullo spazio-tempo, inviate dal gulag al figlio Kirill mediante le lettere del 16 - 17 gennaio e del 3 aprile 1936.

Il presente articolo cercherà di illustrare non tanto gli aspetti teoretici della concezione che Florenskij ebbe della teoria della relatività generale, quanto piuttosto alcuni esempi della ricezione di essa nell'ambito delle sue ricerche e riflessioni sulla complessità empirico-metafisica del reale ${ }^{5}$ - preso in considerazione sia nel suo insieme macrocosmico sia nei singoli elementi

3 Essa, come numerose altre opere di Florenskij, a causa della censura da parte del regime staliniano e della damnatio memoriae cui fu soggetto il suo autore, è stata pubblicata soltanto postuma, prima a Parigi (si è trattato di una pubblicazione non autorizzata dagli eredi), nel 1969, poi, nel 1971, a Tartu.

4 Fa parte dell'opuscolo - di prossima pubblicazione per i tipi di Mimesis Ed. - la spiegazione, a firma di Florenskij, dell'illustrazione (si tratta di una xilografia del pittore V.A. Favorskij) presente sulla copertina, che riassume bene la sua concezione dell'immaginario (la trad. italiana è reperibile in: Florenskij 1990b). Per una presentazione dell'ontogenesi del testo, della spiegazione e dell'illustrazione si veda: Sokolov Pyman 1989. Un'ottima introduzione all'opuscolo, che mette in luce anche la sua non facile ricezione nella Russia sovietica, si trova in: Polovinkin 2010.

5 Si ricorda che Florenskij elaborò tali ricerche e sviluppò tali riflessioni non solo come scienziato (matematico, fisico, chimico, ingegnere elettrotecnico, ecc.), ma prima di tutto come filosofo cristiano e teologo ortodosso. Ne sono una conferma le opere La colonna e il fondamento della Verità (1914), Il significato dell'idealismo (1915), Lezioni sulla concezione cristiana del mondo (1921), Iconostasi (1921 1922), La filosofia del culto (1918 - 1922) e molti altri scritti. Ciò che caratterizza e rende originale il suo approccio filosofico-teologico alla questione della complessità del reale è il riferimento al mistero dell'essere uni-trino di Dio, rivelatosi nella persona di Gesù Cristo, compreso come fondamento (ma anche come idea/energia) che, a partite dal piano della struttura interna, determina ontologicamente e dinamicamente tutto il mondo creato, incluso l'essere umano. Per uno studio più approfondito della fondazione ontologico-trinitaria della teoria florenskijana della complessità si rimanda a: Žák 2016; Burzo 2020. 
microscopici - e in particolare sullo spazio immaginario quale dimensione strutturale di tutto ciò che esiste (cose, oggetti, esseri viventi ecc.). Vista la particolare importanza, per il tema proposto, dell'opuscolo Gli immaginari in geometria, sarà questo il punto di partenza della successiva trattazione.

\section{Einstein, Dante Alighieri e lo spazio immaginario}

Lopuscolo Gli immaginari in geometria fu scritto a più tappe: mentre i paragrafi 1-7 furono redatti nell'agosto del 1902, quando Florenskij studiava alla Facoltà di Matematica e Fisica, gli ultimi due (8-9) furono concepiti venti anni più tardi. Solo nel 1921, infatti, fu aggiunto l'ottavo paragrafo, seguito, nell'anno successivo, dal nono, il conclusivo. Ed è proprio quest'ultimo a includere un riferimento diretto alla teoria della relatività, attraverso una trattazione che interagisce con Einstein a partire da un'originale interpretazione ${ }^{6}$ della descrizione, nel XXXIV canto dell'Inferno dantesco, ${ }^{7}$ di un misterioso fenomeno verificatosi durante la discesa di Dante e Virgilio negli inferi e la loro risalita. Vi si legge che entrambi "mantengono per tutto il tempo della discesa la posizione verticale, con la testa rivolta verso il luogo da cui sono scesi, cioè l'Italia, e i piedi verso il centro della Terra. Quando, però, i due poeti raggiungono indicativamente la cintola di Lucifero, si capovolgono all'improvviso, volgendosi con i piedi verso la superficie della Terra per dove sono entrati nel regno degli inferi, e con il capo nel senso opposto" (Florenskij 1991, 45; 2007, 280-281). E non è tutto: risalendo dalle profondità attraverso un passaggio crateriforme, Dante, asceso al monte del Purgatorio e salito attraverso le sfere celesti, "dopo aver volto lo sguardo da lassù, dall'empireo, verso la Gloria di Dio, si ritrova a Firenze senza essere di fatto tornato indietro” (Florenskij 1991, 46; 2007; 282). È esattamente questo curioso fatto quello che suscita l'interesse di Florenskij: "Dante si muove sempre in linea retta e in cielo sta con i piedi rivolti verso il luogo da cui è disceso"; ebbene, "avendo proceduto sempre in linea retta ed essendosi capovolto una sola volta lungo il cammino, il poeta giunge al luogo di partenza nella stessa posizione che aveva nel momento in cui l'aveva lasciato. Di conseguenza, se strada facendo non si fosse capovolto, lungo la retta egli sarebbe giunto al luogo di partenza a testa in giù" (Florenskij 1991, 46; 2007, 282).

Esaminando il racconto della Divina Commedia, Florenskij giunse a concludere che "la superficie su cui Dante si muove è tale che, con un capovolgimento di direzione, la linea retta che vi si segua porta a un ritorno al punto precedente in posizione eretta, laddove un moto rettilineo, senza inversioni, riporterebbe al punto di partenza un corpo capovolto" (Florenskij 1991, 46; 2007, 282). "Evidentemente" - spiegava il pensatore russo - "si tratta di una superficie che, primo, in quanto contiene rette chiuse, è un piano di Riemann, secondo, in quanto capovolge la perpendicolare che su di essa si muove, è una superficie unilatera. Tali condizioni sono sufficienti a caratterizzare geometricamente lo spazio di Dante come conformato alla geometria ellittica" (Florenskij 1991, 46; 2007, 282).

6 Tra le presentazioni introduttive e gli studi dedicati, in vario modo, a questa interpretazione di Florenskij si consigliano: Paršin 2002, 117-120; Bethea 1996; 2009, 149-166; Sedych 2011; Oppo 2015; Florenskij 2019; di non poco interesse è la presentazione - attenta alle particolarità della traduzione russa della Divina Commedia utilizzata dal Nostro - offerta dal poeta e filosofo Vjačeslav I. Ivanov (Ivanov 1995), per un periodo in stretto contatto con Florenskij.

7 È degno di nota che esattamente l'Inferno della Divina Commedia sia stato preso in esame - seppure solo in riferimento alla questione delle dimensioni spaziali della "realtà sotterranea" descritta dal poeta fiorentino - già dallo scienziato Galileo Galilei; si vedano le sue Due lezioni all'Accademia fiorentina circa la figura, sito e grandezza dell'inferno di Dante, tenute nel 1588: Galileo 1970, 47-80. 
Secondo Florenskij, il fatto descritto da Dante trovava una conferma e un ulteriore approfondimento nella fisica moderna, grazie alla teoria della relatività. Ricorrendo, dunque, al principio della relatività speciale, si poteva dimostrare che non cè esperimento fisico in grado di dar prova del moto della Terra attorno al Sole e che, quindi, il sistema copernicano non è altro che una metafisica "nel senso più deleterio del termine" (Florenskij 1991, 47; 2007, 284). Quanto, invece, alla rotazione della Terra attorno al proprio asse, anch' essa oggetto di indagine da parte di Copernico, la teoria della relatività generale - riportata ad esempio nella formulazione di Philipp Lenard - mostrava che "non ci sono e sostanzialmente non possono esserci prove" (Florenskij 1991, 48; 2007, 285) nemmeno di questo movimento.

$\mathrm{Ma}$, secondo Florenskij, dati ancor più interessanti emergono quando il principio della relatività speciale e quello della relatività generale vengono impiegati con riferimento al principio della velocità della luce: $c=3 \cdot 10^{10} \mathrm{~cm} / \mathrm{sec}$. A suo parere, cioè, la teoria della relatività permette di reinterpretare il concetto di velocità cosmica massima $(=c)$ e di svelare il senso più profondo della concezione della velocità della luce quale "limite ultimo". Perché, si chiede, tale velocità dovrebbe essere considerata un limite ultimo? Presumibilmente, si risponde, per il fatto che il suo superamento implicherebbe la comparsa di condizioni di vita del tutto nuove, trascendenti la nostra esperienza terrena. Tuttavia l'affermare questo non comporta che tali condizioni siano impensabili o inimmaginabili. Il nucleo della questione sta nel riconoscere che "in presenza di velocità pari o - tanto più - superiori a $c$, la vita del mondo è qualitativamente differente da ciò che si osserva con velocità minori a $c$ " e, inoltre, che "il passaggio tra gli ambiti di questa differenziazione qualitativa è pensabile solo come discontinuo" (Florenskij 1991, 49; 2007, 286).

Per immaginare gli effetti di tale ipotizzata nuova situazione (del verificarsi, cioè, della velocità superiore a $c$ ), si deve partire dal presupposto che le

"caratteristiche dei corpi di un sistema in movimento osservato da uno immoto dipendono dal valore essenziale

$$
\beta=\sqrt{1-\frac{v^{2}}{c^{2}}}
$$

dove $v$ è la velocità di movimento del sistema, e $c$ la velocità della luce. Fintanto che $v$ è minore di $c$, di fatto $\beta$ e tutte le altre caratteristiche restano immanenti allesperienza terrena; con $v$ uguale a $c, \beta=0$, e con $v$ maggiore di $c, \beta$ diventa immaginario. In questi due ultimi casi ha luogo un doppio balzo qualitativo delle relative caratteristiche" (Florenskij 1991, 50; 2007, 287).

Nel caso di $\beta=0$ la "lunghezza di qualunque corpo diventa uguale a zero, la massa diventa infinita e il suo tempo - osservato dal di fuori - altrettanto. In altri termini, il corpo perde la propria estensione, diventa eterno e acquisisce una stabilità assoluta" (Florenskij 1991, 50; 2007, 287). Invece nel caso di $v>c$, "il tempo scorre in senso inverso, cosicché l'effetto precede la causa", e ciò comporta che "la lunghezza e la massa dei corpi diventano immaginarie" (Florenskij 1991, 50; 2007, 287). Ci si può figurare, quindi, "come, ridottosi a zero, un corpo irrompe [тело проваливается сквозь] attraverso una superficie dalle coordinate corrispondenti, e rovesciandosi su se stesso [выворачивается через самого себя] acquista caratteristiche immaginarie" (Florenskij $1991,51 ; 2007,287-288)$. In tal caso intervengono infatti condizioni qualitativamente nuove per l'esistenza dello spazio, caratterizzate appunto da parametri immaginari. Questi, tuttavia, sono da considerare come oggettivamente reali, esistenti. Nel senso che "come lo sprofondamento [провалla rottura] della figura geometrica ne determina non la distruzione, bensì un mero passaggio 
all'altro lato della superficie e, di conseguenza, l'accessibilità a esseri che si trovano proprio su questo lato, allo stesso modo l'immaginarietà $[$ мнимость $]$ dei parametri del corpo andrà intesa non quale segno della sua irrealtà, ma come mera testimonianza del suo passaggio a una realtà altra" (Florenskij 1991, 51; 2007, 288).

Florenskij non ha dubbi: l'ambito degli immaginari esiste oggettivamente e fa parte della complessità del reale. ${ }^{8}$ Infatti, ogni spazio è come doppio, "in quanto costituito dalle reali [uз действительных] е - con esse coincidenti [identiche] - immaginarie superfici vettoriali di Gauss, tuttavia il passaggio dalla superficie reale a quella immaginaria è possibile solo attraverso una rottura [через разлом] dello spazio e il rovesciamento [выворачивание] del corpo su se stesso» (Florenskij 1991, 51; 2007, 288).

Florenskij è inoltre convinto che il tema della dualità dello spazio e, quindi, del reale stesso non possa esaurirsi con il discorso sui "due mondi", quello invisibile dell'Empireo - per dirla con Dante - e quello visibile terreno, ${ }^{9}$ dato che quello di immaginario è un concetto complesso che implica innumerevoli stati intermedi (della duplice spazialità). Allo stesso tempo, è persuaso che il superamento della velocità della luce da parte delle particelle del corpo non sia l'unica via possibile per sperimentare la rottura dello spazio e il rovesciamento del corpo su se stesso. Sono esattamente questi i temi di cui il pensatore russo si è occupato, in diversi suoi scritti, facendo riferimento, in modo esplicito o implicito, alla teoria della relatività generale di Einstein.

\section{Lo spazio-tempo e la sua molteplicità}

Cè un presupposto che Florenskij considera della massima importanza per un ulteriore sviluppo del tema degli immaginari e, di conseguenza, del tema della dimensione simultaneamente reale/visibile e immaginaria/invisibile dello spazio. Ne parla persino in una lettera dal gulag, del 16-17 gennaio 1936, in cui spiega che lo spazio-tempo è non solo reale, ma anche assolutamente determinante per i fenomeni naturali. Vi sottolinea che, essendo lo spazio-tempo una "entità" esistente, reale - e quindi non soggettiva né fittizia -, esso agisce; e, in qualità di forma interna, è un fattore fondamentale dei fenomeni naturali. Chiaramente, anche "questa concezione s'intreccia con il principio della relatività" (Florenskij 1998, 369; 2011, 241), incrementando l'interesse per la struttura, dei fenomeni naturali, ovviamente, ma in primis dello stesso spazio-tempo, ${ }^{10}$ e per l'elemento-chiave di essa: la curvatura della superficie.

8 Riferendosi a questi risultati teoretici della trattazione florenskijana, il matematico Renato Betti ne dà la seguente valutazione: "Dal punto di vista matematico, il saggio su Gli immaginari in geometria è una delle opere matematiche più significative di Florenskij, per il suo contenuto che proietta le strutture formali su una concreta interpretazione cosmologica. Il lavoro esplicita un nuovo modello rappresentativo dei numeri complessi corrispondente alla visione globale che Florenskij ha dei fenomeni del mondo e dello spirito. Così, il termine 'immaginario' perde alla fine la propria connotazione tecnica, estendendosi alla prospettiva di ciò che si percepisce quando la vita passa - come può passare - dal visibile all'invisibile. Non si riferisce più soltanto ai numeri, alle loro proprietà e alla loro rappresentazione, ma abbraccia ciò che solo si intuisce, si intravede, si immagina, nel desiderio di comprendere e descrivere quanto non è direttamente percepibile con i sensi. Il campo dei numeri complessi diventa una sorta di zona intermedia fra il reale e l'immaginario, con una totale, e felice, adeguatezza dell'intuizione alla terminologia matematica" (Betti 2009, 95).

9 Cfr. il saggio Empiria ed empirismo (Florenskij 1994, 146-178; 2014, 63-109), il capitolo Due mondi ne La colonna e il fondamento della Verità (Florenskij 1990a, 9-14; 2010, 17-21), oppure la parte introduttiva dell'Iconostasi (Florenskij 1995a, 419ss.; 2008, 19ss.).

10 La spazio-temporalità è un tema di basilare importanza per la teoria della complessità di Florenskij ed è evidente che sul suo approfondimento influì non solo il dialogo con la matematica e la fisica, ma anche 
Il motivo dell'interesse di Florenskij per la struttura e, in particolare, per la curvatura della superficie (dello spazio-tempo) viene esplicitato nella lettera dal gulag del 3 aprile 1936, in cui si ribadisce che la superficie dello spazio-tempo e, di conseguenza, tutte le superfici in senso fisico sono curve, di una curvatura media non uguale a zero. Ė proprio tale curvatura a creare la reale distinzione tra un corpo e altri corpi, e, soprattutto, a determinare l'esistenza di ciò che è esterno e di ciò che è interno, e quindi anche di ciò che sta da una parte della superficie come suo volto reale e di ciò che sta oltre come realtà immaginaria. ${ }^{11}$ Dunque la curvatura della superficie, in quanto configurazione di un determinato spazio-tempo, è da considerarsi una vera e propria forma con un suo peculiare potenziale. Di conseguenza essa crea un campo di forze che determina l'andamento dei fenomeni. Di conseguenza si può dire che "tutti i processi hanno luogo sulla superficie, sul confine tra INTERNO ed ESTERNO, ma" - aggiunge Florenskij - "questo confine è qualcosa di molto più complesso di quanto non appaia a unanalisi sommaria. Con il fatto di penetrare nella profondità del corpo poniamo una nuova superficie di confine, ed è questa poi, e non l'interno del corpo, che studiamo e che è oggetto di ricerca" (Florenskij 1998, 429; 2011, 271).

Nella stessa lettera, però, si trova un'altra importante puntualizzazione, in piena sintonia con la teoria einsteiniana: essendo lo spazio-tempo un insieme inscindibile, è evidente che "la curvatura della superficie non è una curvatura astratta della geometria, bensì una curvatura secondo tutte le coordinate, vale a dire anche secondo quelle del tempo" (Florenskij 1998, 429; 2011, 271). Ciò porta a concludere che l'andamento degli eventi sulle superfici di diversa curvatura è differente e, quindi, che su una determinata superficie il tempo scorre con velocità differente rispetto a unaltra superficie, di diversa curvatura.

In realtà, tali rilievi di Florenskij riprendono le ampie riflessioni sullo spazio-tempo già da lui espresse nell'ambito delle lezioni agli Atelier superiori tecnico-artistici di Stato e contenenti spiegazioni di grande rilievo per il nostro tema, formulate a partire dalla constatazione del superamento della concezione euclideo-kantiana di spazio ad opera della fisica moderna ${ }^{12}$ e grazie soprattutto a entrambi i principi della relatività. In virtù di essi, infatti, è stato possibile stabilire che le caratteristiche dello spazio sono "la finitezza, la non omogeneità, l'anisotropia, la multidimensionalità (cinque dimensioni nella concezione di Eddington), la differenza della sua curvatura dallo zero (diversa inoltre nei diversi punti), la multivocità e, in base a un dato approccio (l'utilizzazione degli immaginari), la non connessione" (Florenskij 2000, 283; 1995b, 228). Ma c’è di

con la filosofia e la teologia. Quest'ultima permise al pensatore russo di sviluppare il concetto di spaziotempo con l'utilizzo delle categorie della metafisica cristiana fondata sul dogma trinitario e impostata in chiave del simbolismo ontologico, un concetto elaborato con la ferma convinzione della centralità del tempo (unitamente allo spazio) nel e per il cristianesimo e del particolare ruolo che esso, in quanto religione rivelata, ha, affinché sia possibile effettuare e praticare un ampio approccio conoscitivo alla spazio-temporalità del reale e alla sua "organizzazione". A parere di Florenskij il cristianesimo ha un interesse molto più elevato, rispetto alle religioni precristiane, per un'esatta organizzazione dello spaziotempo, essendo la coscienza cristiana illuminata dall'Eternità. Per una presentazione e un'analisi filosoficoteologica del concetto florenskijano di spazio-tempo di vedano gli studi: Žák 2000; 2009; Chase 2015.

11 Questa distinzione è in piena convergenza con quella tra il noumeno e il fenomeno, ossia tra le dimensioni noumenica e fenomenica della "cosa" (di un oggetto, un fenomeno, una particella del reale ecc.), che si trova descritta in: Florenskij 1992, 159ss.; 2009, 200ss.

12 Va ricordato che alcuni scienziati iniziano a sostenere l'idea della necessità di rivedere tale superamento (Regge 1994, 200-201), in quanto, secondo i dati dell'attuale ricerca, apparirebbe evidente che lo spazio segua le leggi della geometria euclidea, manifestandosi perciò come uno spazio (un universo) piatto, a curvatura (e a energia) nulla, omogeneo e isotropico (Tonelli 2019, 44-46, 63-64). Si tratta comunque di una posizione che non riesce a smentire teoreticamente le giustificazioni e descrizioni matematiche dello spazio addotte dai sostenitori della geometria non euclidea. 
più: Florenskij insiste sulla necessità di riconoscere che nella nostra quotidiana esperienza di essere parte del reale il concetto di spazio fisico appare intrinsecamente connesso con quello di spazio geometrico e psicofisico.

Parlando dello spazio fisico, geometrico e psicofisiologico, Florenskij intende indicare tre differenti spazi (o proprietà spaziali) che realmente coesistono e quindi prospettare tre simultanei approcci conoscitivi alla spazialità complessa di un unico reale? La risposta va cercata nelle sue lezioni del 1925 sulla spazialità, nelle quali, rifacendosi a Einstein, assieme a W. K. Clifford, H. Poincaré, H. Weyl e A. Eddington, egli ribadisce l'importanza della scoperta della dipendenza delle proprietà spaziali dal cosiddetto campo di forze (Florenskij 2000, 81-146; 1995b, 19-85). E ricorda che già Riemann ha affermato che il fondamento delle relazioni metriche dev'essere cercato in forze coesive che agiscono su di esso; soprattutto riconosce che grazie a Einstein e a Weyl è stato possibile sviluppare tale brillante intuizione matematica, dal significato incomparabilmente più profondo e con un ventaglio di utilizzazioni molto più ampio di quelli solitamente presi in considerazione.

La cosa da tener presente - ed ecco la risposta alla succitata domanda - è che un campo di forze è un insieme di forze molteplici e diverse. Queste, da una parte, hanno una caratteristica comune che le definisce quali forze, per cui si può chiamare forza "tutto ciò che è in grado di agire producendo delle variazioni nelle caratteristiche del reale" (Florenskij 2000, 109; 1995b, 48), in primo luogo le variazioni del grado della curvatura della superficie dello spazio; dallaltra, sono comunque di diverso genere, il che spiega perché "l'attività di ciascuna di esse si manifesta e compare soltanto in presenza di una corrispondente sensibilità degli oggetti al loro influsso e rimane sconosciuta se questa sensibilità manca" (Florenskij 2000, 109; 1995b, 48). ${ }^{13}$ Ciò significa che ogni specifica forza determina la curvatura di uno specifico spazio ad essa corrispondente, ma anche che, se esistono determinate forze, devono esserci realmente i rispettivi spazi, esistenti all'interno di un unico reale.

Ma di quali forze si tratta? Florenskij cita la forza di gravità, la forza d'urto, la forza magnetica e la forza della luce, appartenenti al genere di forze di cui si occupa la fisica, e ad esse aggiunge la forza dell'attenzione, la forza dell'impressione, la forza della passione, la forza della bellezza, la forza dello spettacolo e la forza della parola, facendo intuire che questo elenco potrebbe essere molto più lungo (Florenskij 2000, 104-110; 1995b, 43-48). Pertanto anche l'odorato, il gusto, le emozioni mistiche di diverso genere, i pensieri e persino le sensazioni hanno delle caratteristiche spaziali, il che vuol dire che sono anch'essi determinati da forze corrispondenti. Sta di fatto che ogni genere di forza crea, con caratteristiche peculiari e differenti, una determinata curvatura di una specifica superficie spaziale.

Eppure Florenskij, appena ribadendo che, essendoci molte forze, ci sono di conseguenza molti spazi, insiste sulla necessità di considerare il reale - e di descriverlo, creandone modelli conoscitivi - come un insieme la cui multispazialità non significa disgregazione ma interconnessione e coordinazione. Sicuramente, all'interno di un unico reale (di un'unica "cosa") intrinsecamente multispaziale si verifica il fenomeno dell'essere-fuori-luogo (внеположность), apparendo certi elementi esterni rispetto ad altri a causa del loro posizionamento in un proprio spazio, un posizionamento differente rispetto a quello degli elementi degli altri spazi di un unico reale. Tuttavia, pur riconoscendoli come esterni e pur differenziandoli, si è in grado di cogliere i differenti elementi come un insieme, come un qualcosa di interconnesso e coordinato, il che porta

13 "Fra le diverse forze non cè una frontiera divisoria, da un lato della quale si trovi ciò che è oggettivo e dall'altro ciò che è soggettivo. Ciò che è oggettivo possiede il suo proprio lato interiore, allo stesso modo in cui ciò che è soggettivo può venire svelato. Non cè niente di misterioso che non divenga evidente e, viceversa, tutto ciò che è evidente nasconde in sé un mistero" (Florenskij 2000, 109; 1995b, 48). 
a riconoscere il reale quale un insieme organico, un'unità differenziata e una molteplicità unita (Florenskij 2000, 110-111; 1995b, 48-49).

L'argomentazione addotta mette bene in luce la grande complessità del concetto che Florenskij ha di spazialità simultaneamente geometrica, fisica e psicofisica, assieme al motivo di tale triplice strutturazione. Egli è convinto che la comprensione e la descrizione dello spazio da parte della geometria o della fisica - dell'"alleanza" delle quali non si può dubitare - non debbano scindersi dall'esperienza concreta del reale, garantita in particolare dalla sua percezione psicofisica, più idonea della fisica e della geometria a recepire e descrivere esaustivamente l'interna complessità dei campi di forze che determinano lo spazio.

L'originalità di tale concezione di Florenskij emerge ancor di più quando si prendono in considerazione gli ulteriori sviluppi della sua riflessione. Mi riferisco, ad esempio, alla sua idea che la spazialità (i differenti spazi e le loro curvature) esista come una realtà non solo data a priori, e con la quale bisogna fare i conti per il fatto di essere venuti all'esistenza all'interno di un ambiente reale già esistente, ma anche determinabile dagli esseri umani, essendo questi in grado di modificare gli spazi esistenti e, addirittura, di ricrearne di nuovi, e di stabilire una forza, o un determinato campo di forze, che, di conseguenza, crei una determinata curvatura della superficie di un determinato spazio. Mi riferisco inoltre alla convinzione - elaborata sul terreno della teoria della relatività - che Florenskij sviluppò a partire dalla concezione dello spazio e del tempo come di due realtà differenti eppure intrinsecamente unite. Essa poggia sul presupposto che la molteplicità degli spazi coincide con la molteplicità dei tempi, dato che la velocità del tempo (di una sequenza di attimi di tempo) dipende dal grado della curvatura della superficie sulla quale un determinato corpo si muove. Dunque, se esistono differenti forze che creano differenti curvature della superficie - creando in questo modo differenti spazi -, devono esistere anche tempi che scorrono con differenti velocità. Tutto questo caratterizza la complessità di un unico reale, sia nel suo insieme che nei suoi particolari.

Ebbene, è in questa prospettiva che deve essere interpretata l'idea di Florenskij circa la possibilità della rottura di una superficie e del riversarsi (rovesciarsi) su se stesso di un corpo che con ciò - per chi si trovava con esso da una parte della superficie di un determinato spazio - diventa immaginario, acquisendo delle caratteristiche nuove, corrispondenti a quelle che si verificano con il superamento della velocità della luce.

\section{Predisposizione dell'essere umano all'esperienza dell'immaginario}

Nel testo di una lezione di Florenskij del 1924, appuntata da una studentessa, si legge: «Il tempo scorre sempre in una nuova direzione che ha un unico verso e, in un significato preciso e sostanziale, è irreversibile. Questa è l'opinione comune sul tempo e sullo spazio. Cercherò ora di dimostrare che tale opinione è sbagliata» (Florenskij 2000, 371; 1995b, 317).

È sbagliata, perché la concezione del tempo non deve limitarsi al "tempo della fisica", ma deve prendere in considerazione anche il "tempo psicofisico". Ne è una riprova la realtà del sogno, che Florenskij cita nel testo della lezione e della quale si occupa ampliamente soprattutto nell'Iconostasi. Nelle prime pagine di questopera, riferendosi all'idea del tempo istantaneo di K. du Prel e alle ricerche di F.W. Hildebrandt, prima di tutto egli spiega che il contesto spazio-temporale in cui sorgono le immagini oniriche è la linea di confine tra sonno e veglia. Esse sorgono come passaggio istantaneo da una sfera all'altra della vita psichica e, solo dopo, alla sfera della memoria, cosicché, pur trasposte nella coscienza diurna e pur sviluppandosi nell'ordine del succedersi temporale proprio del mondo visibile, possiedono "una propria misura del tempo, quella 'trascendentale', non paragonabile a quella diurna" (Florenskij 1995a, 420; 2008, 
20). Dunque, il sogno permette di passare con la coscienza ad un altro sistema, avente una nuova misura del tempo, differente da quella del sistema precedente.

Secondo Florenskij, pur non conoscendo il principio della relatività, quasi tutti gli uomini, grazie all'esperienza elementare del sogno, sono pronti a riconoscere senza difficoltà che in ciascuno dei differenti sistemi scorre un proprio tempo alla propria velocità e con la propria misura. Solo pochi, però, avvertono un altro fatto, ancora più sorprendente: che il tempo del sogno può scorrere a una velocità infinita e, addirittura, voltandosi su se stesso a velocità infinita, può invertire la direzione del suo corso. Questo sta a dimostrare che il tempo può essere "realmente istantaneo e teleologico, rivolto cioè dal futuro al passato, dalle conseguenze alle cause, il che si verifica proprio quando la nostra vita passa dal visibile all'invisibile, dal reale all'immaginario" (Florenskij 1995a, 420; 2008, 20). All'origine di tutto questo può essere comunque uno stimolo esterno, che però viene elaborato e assimilato in base a due coscienze: quella diurna e quella notturna. Una voce esterna, il tocco della fredda spalliera del letto di ferro o altre sollecitazioni esterne riescono, cioè, a provocare un immediato risveglio, che però viene vissuto dal dormiente come un susseguirsi di numerose e lunghe sequenze di eventi, alla cui fine - e solo alla fine! - la stessa causa del risveglio appare in forma simbolica come punto conclusivo della storia vissuta in sogno (Florenskij 1995a, 421-426; 2008, 21-27). ${ }^{14}$

Seè dunquevero che grazieal sogno-questa elementare e universalmente umana esperienza della grande complessità del reale - è possibile irrompere nella spaziotemporalità degli immaginari, il significato più profondo di un simile rovesciamento su se stessi è da ricercare, secondo Florenskij, nell'orizzonte della fede religiosa, in particolare quella cristiana, dotata di appositi concetti e di adeguate descrizioni. Egli spiega:

"Forse in questo mondo rovesciato, in questo riflesso ontologicamente speculare del mondo, non riconosciamo la sfera dell'immaginario, sebbene questo immaginario, per coloro che si sono rivoltati su se stessi e si sono rovesciati arrivando fino al centro spirituale del mondo, sia anch'esso autenticamente reale esattamente come loro. Sì, questo immaginario è reale nella sua essenza, non è qualcosa di totalmente diverso rispetto a questo nostro mondo. Essendo la benefica creazione di Dio una realtà unica, ciò che viene guardato dall'altro lato, da parte di chi vi si trova, è sempre la stessa e identica realtà. Da lì si vedono i sembianti e gli sguardi spirituali delle cose, visibili a chi ha reso manifesto in sé il proprio sembiante originario, l'immagine di Dio, ossia, per dirla in greco, l'idea: le idee della realtà esistente vedono coloro che, dalle idee, sono interamente illuminati, manifestando in sé e attraverso sé stessi, a questo nostro mondo, le idee del mondo celeste" (Florenskij 1995a, $427 ; 2008,28)$.

Il sogno non rappresenta, certo,l'unica modalità di entrata nella spaziotemporalità dell'immaginario. Anche le creazioni artistiche, ad esempio, permettono una simile esperienza, seppure di altro genere, avendo al centro l'idea creativa/ispiratrice di una concreta opera artistica. Pertanto, sia il sogno che le opere d'arte - e la loro creazione - testimoniano l'esistenza di un preciso dato di fatto: la strutturale predisposizione dell'essere umano a fare l'esperienza dell'immaginario. Forte di questa convinzione, Florenskij afferma:

${ }_{14}$ Ad esempio, un tocco della fredda spalliera sul collo nudo fa sognare una lunga e drammatica storia che finalmente si conclude, coincidendo con il risveglio del dormiente, quando il coltello della ghigliottina ne sfiora il collo. 


\begin{abstract}
“Sì, la vita della nostra stessa anima [душа - significa anche 'psyché] offre il punto di appoggio per poterci esprimere su questa linea di contatto tra i due mondi, poiché anche in noi stessi il vivere nel visibile si alterna con il vivere nell'invisibile. E perciò ci sono momenti - seppur brevi, ridotti, a volte della durata di un solo istante - in cui i due mondi si toccano e noi possiamo contemplarne il contatto. Dentro di noi per alcuni istanti si squarcia il velo dell'invisibile e attraverso il suo strappo, ancora percepibile, soffia l'alito invisibile di altri luoghi: i due mondi si sciolgono l'uno nell'altro e la nostra vita diventa una corrente continua, come quando nell'afa l'aria calda sale in alto" (Florenskij 1995a, 419; 2008, 19).
\end{abstract}

Sembrerebbe che le parole di Florenskij facciano parte di un discorso intenzionato a sostenere l'idea di un'intrinseca predisposizione umana a quel tipo di esperienze particolari di cui si occupano esclusivamente la teologia mistica o gli studi del paranormale. Non è esattamente così; perlomeno questa impressione non coglie tutta la verità sull'anima come luogo dell'incontro tra i due mondi. Infatti, Florenskij - sempre fermamente convinto dellimportanza della religione e della mistica per la conoscenza del reale - si riferisce qui in primo luogo alla strutturale predisposizione conoscitiva propria di tutti gli esseri umani, che, se presa seriamente in considerazione, è di cruciale importanza per la loro esperienza conoscitiva del reale. Volendo richiamare l'attenzione su questo tema persino in una lettera dal gulag (del 21 febbraio 1936), egli scrive al figlio Kirill:

"Il principio (minore) della relatività si basa semplicemente sul carattere necessario di ciò che tutti conoscono, ma con cui nessuno fa i conti nella vita concreta. In parole povere, per il fatto di vivere noi entriamo in comunicazione o ammettiamo la sua possibilità direttamente, non attraverso lo spazio, ma al di sopra dello spazio [fisico], oltrepassando le percezioni. [...] Occorre riconoscere che è veramente possibile una comunicazione con mezzi non fisici, o su un terreno non fisico: deve allora essere ricostruita quella teoria della conoscenza cui si attiene la maggioranza delle persone, deve essere ricostruita dalla base e, anche in tal caso, con tutte le conseguenze che ne derivano. Noi conosciamo una cosa non perché la vediamo, la udiamo, la fiutiamo e la tocchiamo, ma al contrario: se vediamo, udiamo, fiutiamo e tocchiamo una cosa, è perché l'abbiamo già conosciuta prima, cogliendola - anche se inconsciamente o al di sopra della coscienza - nella sua autenticità e nella sua realtà diretta. La percezione, allora, deve essere considerata solo come materiale per il trasferimento della cosa dalla sfera inconscia a quella cosciente, e non come materiale del contenuto stesso della conoscenza. Per me ciò è del tutto chiaro, ma non so se sia riuscito a spiegarti l'essenza della questione" (Florenskij 1998, 671; 2011, 379).

\title{
5. Conclusione: la fisica a servizio di una "nuova" Weltanschauung
}

La questione della complessità del reale, e quindi anche degli immaginari, è stata al centro dell'attenzione di Florenskij sin dal tempo dei suoi studi universitari, soprattutto a partire dall' iscrizione all'Accademia teologica di Mosca, da lui decisa nel 1904, dopo una brillante conclusione del ciclo di studi nella Facoltà di Matematica e Fisica presso l'Università di Mosca. Ne è una conferma la breve introduzione alla sua traduzione in russo della Monadologia fisica di I. Kant, scritta nel 1905 (Florenskij 1994, 682-686). L'allora studente di teologia vi tesse la lode della teoria degli insiemi (die Mengenlehre) elaborata da Georg Cantor, prospettata come strumento conoscitivo capace di cogliere, e tenere dialetticamente o dinamicamente insieme, non solo l'unità e la molteplicità dell'essere 
di una cosa, ma anche il suo essere rivolto contemporaneamente verso l'esterno e l'interno, e quindi il suo "apparire" e la sua interioritas, in cui si cela ciò che di essa è "immaginario". Per abbracciare la totalità dell'essere (di una cosa, di un oggetto di conoscenza) occorre perciò ammettere il realismo anche della sua interioritas, inclusi i suoi immaginari, il che spinge a compiere un radicale passaggio dalla visione materialista del mondo a quella spirituale.

Secondo il giovane Florenskij, gli stessi studi fisici sullatomo - quale "centro delle forze" invitavano a fare una scelta di tal genere, tanto più alla luce del fatto che le ricerche iniziavano a focalizzarsi sull'elettrone, una particella subatomica del tutto particolare perché né materiale né fittizia e tuttavia centrale per la costituzione della materia. In questo modo la fisica avrebbe operato una "dematerializzazione della materia" (Florenskij 1994, 686) e così avrebbe invitato l'umanità ad aprirsi ai nuovi fatti e alle nuove idee, per giungere a una visione più ampia e globale del reale. Immergendosi nello studio della teologia e della mistica cristiana e ricevendo, più tardi, la consacrazione sacerdotale, Florenskij intendeva mostrare la validità di un percorso di vita e di conoscenza che non si discostava da tale visione, né la distorceva o frammentava, ma l'arricchiva di un'idea del reale e di un "pensiero complesso" possibili solo a chi vivesse una viva esperienza di fede nel Dio unitrino, ${ }^{15}$ Fondamento trascendente assolutamente "complesso" e infinitamente "semplice" dell'intero universo e di tutti gli esseri.

Non a caso, sempre nel 1904, egli pubblicò un articolo, intitolato Su un presupposto della concezione del mondo, in cui ribadisce con molta convinzionel'idea dell'assenza delle contraddizioni di fondo tra l'ambito dell'osservazione scientifica (la scienza) e quello delle esperienze spirituali/ mistiche (la religione), spiegando che entrambi sono ugualmente necessari all'uomo, essendo essi «ugualmente validi e sacri» per la «realizzazione del Bene» (Florenskij 2007c, 14).

\section{REFERENCES}

Bethea, David M. 1996. Florensky and Dante. Revelation, Orthodoxy, and Non-Euclidean Space. In Kornblatt, Judith D. - Gustafson Richard F. (eds.). Russian Religious Thought. Madison London, 112-131.

Bethea, David M. 2009. The Superstitious Muse. Thinking Russian Literature Mythopoetically. Brighton.

Betti, Renato. 2009. Pavel Florenskij: matematica e visione del mondo. In Emmeciquadro 35, 83-96.

Burzo, Domenico. 2018. Sul limite del crepuscolo. L'intuizione platonica nel pensiero di Pavel A. Florenskij. Roma.

Burzo, Domenico. 2020. La conversione di un uomo moderno. Pavel Florenskij e il sentiero dell'esperienza religiosa. Milano - Udine.

Florenskij, Pavel A. 1990a. Столп и утверждение Истины. Опыт православной теодицеи в двенадцати письмах [The Pillar and Ground of the Truth: An Essay in Orthodox Theodicy in Twelve Letters], vol. 1. Moscow [la prima ed. è del 1914].

Florenskij, Pavel A. 1990b. Spiegazione della copertina. In Florenskij, Pavel A. La prospettiva rovesciata e altri scritti, trad. dal russo a cura di N. Misler. Roma, 136-143.

Florenskij, Pavel A. 1991. Мнимости в геометрии. Расширение области двухмерных образов геометрии [Imaginary Points in Geometry. The Expansion of the Domain of Two-Dimensional Images in Geometry]. Moscow [la prima ed. è del 1922].

${ }_{15}$ Per una conoscenza più approfondita della teologia trinitaria di Florenskij rimango tuttora valide e da raccomandare le monografie: Silberer 1984; Žák 1998. 
Florenskij, Pavel A. 1992. Детям моим; Воспоминанья прошлых дней; Генеалогические исследования; Из соловецких писем; Завещание [For My Children; Memories of Bygone Days; Genealogical Studies; From Solovetsky Letters; Testament]. Moscow.

Florenskij, Pavel A. 1994. Сочинения в четырех томах [Selected Works in 4 Volumes], vol. 1. Moscow.

Florenskij, Pavel A. 1995a. Сочинения в четырех томах [Selected Works in 4 Volumes], vol. 2. Moscow.

Florenskij, Pavel A. 1995b. Lo spazio e il tempo nell'arte, trad. dal russo di N. Misler. Milano.

Florenskij, Pavel A. 1998. Сочинения в четырех томах [Selected Works in 4 Volumes], vol. 4: Письма с Дальнего Востока и Соловков [Letters from the Far East and Solovki]. Moscow.

Florenskij, Pavel A. 2000. Собрание сочинений. Статьи и исследования по истории и философии искусства и археологии [Collected works. Articles and studies on the history and philosophy of art and archeology]. Moscow.

Florenskij, Pavel A. 2007a. Avtoreferat (Nota autobiografica). In Florenskij, Pavel A. Il simbolo e la forma. Scritti di filosofia della scienza, trad. dal russo a cura di N. Valentini e A. Gorelov. Torino, 4-12.

Florenskij, Pavel A. 2007b. Gli Immaginari in geometria [il §9]. In Florenskij, Pavel A. Il simbolo e la forma. Scritti di filosofia della scienza, trad. dal russo a cura di N. Valentini e A. Gorelov. Torino, 278-288.

Florenskij, Pavel A. 2007c. Su un presupposto della concezione del mondo. In Florenskij, Pavel A. Il simbolo e la forma. Scritti di filosofia della scienza, trad. dal russo a cura di N. Valentini e A. Gorelov. Torino, 13-24.

Florenskij, Pavel A. 2008. Iconostasi. Saggio sullicona, trad. dal russo a cura di G. Giuliano. Milano. Florenskij, Pavel A. 2009. Ai miei figli. Memorie di giorni passati, trad. dal russo a cura di N. Valentini e L. Žák. Milano.

Florenskij, Pavel A. 2010. La colonna e il fondamento della Verità, trad. dal russo a cura di N. Valentini. Cinisello Balsamo. Milano.

Florenskij, Pavel A. 2011. "Non dimenticatemi». Le lettere dal gulag del grande matematico, filosofo e sacerdote russo, trad. dal russo a cura di N. Valentini e L. Žák. Milano.

Florenskij, Pavel A. 2014. Il cuore cherubico. Scritti teologici, omiletici e mistici, trad. dal russo a cura di N. Valentini e L. Žák. Cinisello Balsamo.

Florenskij, Pavel V. 2019. Трансформация Космоса: от Данте - или к иконе или к атомной бомбе [The transformation of the cosmos: from Dante - to the icon or to the atom bomb]. In Bertelé, Matteo (ed.). Pavel Florenskij tra Icona e Avanguardia. Venezia, 147-159.

Galileo, Galilei. 1970. Scritti letterari, a cura di A. Chiari. Firenze.

Guerrisi, Marina. 2017. Caleidoscopi e conchiglie. Introduzione alla simbolica di Pavel A. Florenskij. Milano.

Chase, Michael. 2015. Pavel Florensky on Space and Time. In Schole 9/1, 105-118.

Ivanov, Vjačeslav. 1995. П.А. Флоренский и проблема языка [Р.A. Florensky and the problem of language]. In Hagemeister, Michael - Kauchtschischwili, Nina (eds.), P.A. Florenskij e la cultura della sua epoca, Marburg, 214-223.

Oppo, Andrea. 2015. Se la misura di un corpo è un numero immaginario. Florenskij e il concetto di spazio in Dante. In Theologica \& Historica 24, 171-183.

Paršin, Aleksej N. 2002. Путь. Математика и другие миры [The Way. Mathematics and other worlds]. Moscow.

Polovinkin, Sergej M. 2010. Реальность 1920-1930-х годов и «Мнимости геометрии» священника Павла Флоренского [The reality of the 1920s-1930s and the «Imaginary Points in Geometry» by priest Pavel Florensky]. In Polovinkin, Sergej. Русская религиозная 
философия: Избранные статьи [Russian Religious Philosophy: Selected Articles]. St. Petersburg, 68-85.

Regge, Tullio. 1994. Infinito. Viaggio ai limiti dell'universo. Milano.

Silberer, Michael. 1984. Die Trinitätsidee im Werk von Pavel A. Florenskij. Versuch einer systematischen Darstellung in Begegnung mit Thomas von Aquin. Würzburg.

Sedych, Oksana M. 2011. Архаический космос и современная наука [Archaic space and modern science]. In Человек [Human] 6, 152-170.

Sokolov, Kirill - Pyman, Avril. 1989. Father Pavel Florensky and Vladimir Favorsky: Mutual Insight into the Perception of Space. In Leonardo 22/2, 237-244.

Tagliagambe, Silvano. 2006. Come leggere Florensky. Milano.

Tagliagambe, Silvano - Spano, Massimiliano - Oppo, Andrea (eds.). 2018. Il pensiero polifonico di Pavel Florenskij. Una risposta alle sfide del presente. Cagliari.

Tonelli, Guido. 2019. Genesi. Il grande racconto delle origini. Milano.

Žák, Lubomír. 1998. Verità come ethos. La teodicea trinitaria di P. A. Florenskij. Roma.

Žák, Lubomír. 2000. Il mistero del tempo come "quarta dimensione" in Pavel Florenskij. In Filosofia e Teologia 14/1, 49-64.

Žák, Lubomír. 2009. Lunità e la molteplicità dello spazio e del tempo secondo la teoria della discontinuità di Pavel Aleksandrovič Florenskij. In Alfano, Marina - Buccheri, Rosolino (eds.), Tempo della Fisica e Tempo dell'Uomo: Relatività e Razionalità, Atti della seconda giornata di studio del ciclo di incontri annuali "Apeiron: scienza-filosofia-religione". Trapani-Ferrara, 191-226.

Žák, Lubomír. 2016. La complessità del reale e la sua conoscenza. Spunti di riflessione sull'allargamento della ragione proposto da P.A. Florenskij. In Divus Thomas 119/3, 137-171.

Žák, Lubomír (ed.). 2017. Pavel A. Florenskij: "Ho contemplato il mondo come un insieme". Teologia, filosofia e scienza di fronte alla complessità del reale. Città del Vaticano (numero monografico di Lateranum 83/3, 511-696).

prof. ThDr. L'ubomír Ž́ák

Pontificia Università Lateranense

00120 Città del Vaticano

Vatican City State

zak@pul.va

Palacký University Olomouc

Sts Cyril and Methodius Faculty of Theology

Univerzitní 22

77111 Olomouc

Czech Republic

lubomir.zak@upol.cz 